\title{
THE CANTOR TREE, THE $\gamma$-PROPERTY, AND BAIRE FUNCTION SPACES
}

\author{
DANIEL K. MA
}

(Communicated by Franklin D. Tall)

\begin{abstract}
Let $X \subseteq 2^{\omega}$ and $T \cup X$ be the Cantor tree over $X$. We show that $C_{k}(T \cup X)$ is a Baire space if and only if $X$ is a $\gamma$-set. We obtain from this result consistent examples of spaces $Y$ and $Z$ such that $C_{k}(Y)$ and $C_{k}(Z)$ are Baire spaces but $C_{k}(Y) \times C_{k}(Z)$ is not a Baire space. It also follows that there are consistent examples of locally compact nonparacompact spaces $Y$ such that $C_{k}(Y)$ is Baire but not weakly $\alpha$-favorable.
\end{abstract}

\section{INTRODUCTION}

This paper will show that the Cantor tree and the $\gamma$-property are useful in providing insight on the Baire property of function spaces with the compactopen topology. Specifically, we consider function spaces on the Cantor tree. All spaces under consideration are Tychonoff, and our notation and terminology, if not defined, can be found in [8, 17].

Definition 1.1. Let $Y$ be a space, $A \subseteq Y$, and $U \subseteq \mathbb{R}$. Denote the set of all continuous real-valued functions on $Y$ by $C(Y)$, and let $[A, U]=\{f \in$ $C(Y): f(A) \subseteq U\}$.

The collection $C(Y)$ can be endowed with the pointwise convergence topology and the compact-open topology. The former topology is generated by the subbase

$$
\{[A, U]: A \subseteq Y \text { is finite and } U \subseteq \mathbb{R} \text { is an open interval }\},
$$

and $C(Y)$ with this topology is denoted by $C_{p}(Y)$. The latter topology is generated by the subbase

$$
\{[A, U]: A \subseteq Y \text { is compact and } U \subseteq \mathbb{R} \text { is an open interval }\},
$$

and $C(Y)$ with this topology is denoted by $C_{k}(Y)$.

Received by the editors October 23, 1991 and, in revised form, March 24, 1992; this paper has been presented at the 26th Annual Spring Topology Conference sponsored by NSF and the University of North Carolina at Charlotte, April 2, 1992. Parts of this paper have also been presented at the AMS annual meeting held in San Antonio, TX.

1991 Mathematics Subject Classification. Primary 54C35, 54E52; Secondary 54A35.

This paper represents part of the author's doctoral dissertation, Auburn University, under the direction of Professor Gary Gruenhage. 
In [13] the Baire property of $C_{p}(Y)$ is characterized in terms of some topological property of the domain space $Y$. Because this property of $Y$ is preserved under free unions, it follows that the Baire property of $C_{p}(Y)$ is productive, i.e., $\prod_{\alpha \in S} C_{p}\left(X_{\alpha}\right)$ is a Baire space if $C_{p}\left(X_{\alpha}\right)$ is a Baire space for each $\alpha$ in $S$. In this paper, we will show that it is consistent with the usual (ZFC) axioms of set theory that there are Baire function spaces $C_{k}(Y)$ and $C_{k}(X)$ such that $C_{k}(X) \times C_{k}(Y)$ is not Baire. We do not know if such examples exist in ZFC without using extra axioms.

Remark. The function space $C_{k}(Y)$ is a topological vector space (with the pointwise addition of functions and the pointwise scaler multiplication of functions) and is also a topological group (with the pointwise addition). In [15] van Mill and Pol obtained Baire subspaces of some topological vector spaces (topological groups) with non-Baire product. Their construction was based on the direct construction of two Baire spaces with non-Baire product by Fleissner and Kunen [1].

While we do not know of a characterization for the Baire property of $C_{k}(Y)$ in terms of a property on $Y$, we can piece together, from [6,11], a characterization for a stronger completeness property of $C_{k}(Y)$, namely, the property of being weakly $\alpha$-favorable (see $\S 2$ ). We have the following theorem (see $\S 2$ for the proof).

Theorem 1.2. For any locally compact space $Y, C_{k}(Y)$ is weakly $\alpha$-favorable if and only if $Y$ is paracompact.

We will provide, under a certain set-theoretic hypothesis, a locally compact nonparacompact space $Y$ such that $C_{k}(Y)$ is Baire. Furthermore, one such $Y$ is normal and another such $Y$ is not normal. Thus, for locally compact spaces $Y$, the Baire property of $C_{k}(Y)$ is consistently weaker than that of being weakly $\alpha$-favorable.

Remark. The fact that the Baire property of $C_{k}(Y)$ is weaker than that of being weakly $\alpha$-favorable is known for general spaces $Y$. Let $Y=\omega \cup\{q\}$, where $\omega$ is the space of all natural numbers and $q \in \beta \omega$, and $Y$ has the topology inherited from $\beta \omega$, the Stone-Čech compactification of $\omega$. It was shown in $[10,13]$ that $C_{p}(Y)$ is a Baire space but not weakly $\alpha$-favorable. It is true that for any space $Z$, if $C_{p}(Z)$ is Baire, then $C_{k}(Z)=C_{p}(Z)$. Thus, $C_{k}(Y)=C_{p}(Y)$ is Baire but not weakly $\alpha$-favorable. Note that $Y=\omega \cup\{q\}$ is not locally compact.

The domain spaces $Y$ of our examples of $C_{k}(Y)$ are subsets of the Cantor tree. As before, $\omega$ is the set of all natural numbers, and we identify a natural number $n$ with the set $\{k \in \omega: k<n\}$. We further identify $2^{\omega}\left(2^{n}\right)$ with the set of all functions from $\omega(n)$ to $2=\{0,1\}$. We use the fact that $C=2^{\omega}$, as a product space, is the Cantor set. Let $T=\bigcup_{n<\omega} 2^{n}$.

Definition 1.3. The Cantor tree is the set $T \cup C$ with elements of $T$ considered isolated and points in $C$ having basic open sets of the form

$$
[x \mid n, x]=\{x \mid j: j \in \omega \text { and } j \geq n\} \cup\{x\}
$$

where $n<\omega, x \in C$, and the symbol " $\mid$ " in $x \mid n$ is our symbol for the restriction of functions. We will refer to the set $[x \mid n, x]$ as the branch for $x$ below the $n$th level in the Cantor tree. 
We consider subsets of the Cantor tree of the form $T \cup X$ where $X \subseteq C$. Note that $T \cup X$ is a locally compact space. Our main result is that $C_{k}(T \cup X)$ is a Baire space if and only if $X$ is a $\gamma$-set. We will use uncountable $\gamma$-sets (one can be of cardinality continuum) under Martin's axiom (MA) [3]. Every $\gamma$-set is of strong measure zero [3, 4]. Hence, in Laver's model [9] for the Borel conjecture there are no uncountable $\gamma$-sets. There are also two subsets of the real line that are $\gamma$-sets, in some models of set theory, whose union is not a $\gamma$-set. This was proved by Todorcevic (quoted in [2,3]). We will appeal to these models to obtain our examples.

Remark. There is an interesting connection between $C_{k}(T \cup X)$ and $C_{p}(X)$. In [5] Gerlits proved that $C_{p}(X)$ is a Fréchet-Urysohn space (also a $k$-space) if and only if $X$ has the $\gamma$-property. Thus, $C_{k}(T \cup X)$ is a Baire space if and only if $X$ is a $\gamma$-set if and only if $C_{p}(X)$ is a Fréchet-Urysohn space.

\section{BACKGROUND RESULTS AND RELATED DEFINITIONS}

Definition 2.1. Let $Y$ be a space and $\mathscr{A}$ be a collection of nonempty compact subsets of $Y$. The collection $\mathscr{A}$ is a moving-off collection for $Y$ means that, for each compact subset $K$ of $Y$, there is some $A \in \mathscr{A}$ such that $A \cap K=\varnothing$. A collection of subsets of $Y, \mathscr{B}$, has a discrete open expansion means that for each $B \in \mathscr{B}$ there is an open set $O(B) \supseteq B$ such that $\{O(B): B \in \mathscr{B}\}$ is a discrete family in $Y$. The space $Y$ has the moving off property (MOP) means that each moving-off collection for $Y$ has an infinite subcollection that has a discrete open expansion.

In [12, Corollary 5.3.6] the following theorem was proved.

Theorem 2.2. If $C_{k}(Y)$ is a Baire space, then $Y$ has the MOP.

The Baire property has a game characterization, and the game can be used to define the property of being weakly $\alpha$-favorable mentioned in $\S 1$. Two players, the empty set $(\mathrm{E})$ and the nonempty set $(\mathrm{NE})$, play the Banach-Mazur game on the space $Y$, denoted by $\operatorname{BM}(Y)$. To start off, $E$ chooses a nonempty open subset of $Y, U_{0}$, and NE chooses a nonempty open subset $V_{0} \subseteq U_{0}$. At the $n$th move $(n>0), E$ chooses a nonempty open set $U_{n} \subseteq V_{n-1}$ and NE chooses a nonempty open set $V_{n} \subseteq U_{n}$. The player NE wins if and only if $\bigcap_{n<\omega} V_{n} \neq \varnothing$; otherwise, player $\mathrm{E}$ wins.

Remark. It is known [7] that $Y$ is a Baire space if anad only if the player E has no winning strategy in the game $\operatorname{BM}(Y)$. If we modify the game by letting NE make the first move, then it is true [7] that $Y$ is of second category if and only if $\mathrm{E}$ has no winning strategy in the modified game $\mathrm{BM}(Y)$.

A weakly $\alpha$-favorable space is one in which the nonempty player NE has a winning strategy in the Banach-Mazur game on that space. We now prove Theorem 1.2.

Proof of Theorem 1.2. $(\Leftarrow)$ In [12] it was shown that $Y$ is a locally compact paracompact space implies that $C_{k}(Y)$ is a Baire space. In fact, the same argument shows that $C_{k}(Y)$ is weakly $\alpha$-favorable since $C_{k}(Y)$ was shown to be homeomorphic to a product of completely metrizable spaces. Note that any complete metric space is weakly $\alpha$-favorable (the player NE chooses his/her 
open sets with diameters going to zero) and a product of weakly $\alpha$-favorable spaces is weakly $\alpha$-favorable [16].

$(\Rightarrow)$ In [11] McCoy and Ntantu showed that $C_{k}(Y)$ is weakly $\alpha$-favorable implies that player II has a winning strategy in the game $\Gamma_{1}(Y)$, which is defined as follows (the game is played by two players, I and II):

To start off, player I chooses a compact subset $A_{0}$ of $Y$ and player II chooses a compact subset $B_{0}$ of $Y$. At the $n$th stage $(n>0)$, player I chooses a compact subset $A_{n}$ of $Y$ such that $A_{n} \cap\left(B_{0} \cup \cdots \cup B_{n-1}\right)=\varnothing$ and player II chooses a compact subset $B_{n}$ of $Y$. Player II wins if and only if $\left\{A_{n}: n<\omega\right\}$ is a discrete family in $Y$; otherwise, player I wins.

In [6] Gruenhage showed that for a locally compact space $Y, Y$ is paracompact if and only if $K$ has a winning strategy in the game $G^{*}(Y)$, which is played by two players $K$ and $L$ and is defined as follows:

At the $n$th play, $K$ chooses a compact subset $K_{n}$ of $Y$, and $L$ chooses a compact subset $L_{n}$ of $Y$ such that $L_{n} \cap K_{n}=\varnothing . K$ wins if and only if $\left\{L_{n}: n<\omega\right\}$ is a locally finite family in $Y$. We claim the following:

Claim. For a locally compact space $Y$, player II has a winning strategy in the game $\Gamma_{1}(Y)$ implies $K$ has a winning strategy in the game $G^{*}(Y)$.

Proof of Claim. Let $\sigma$ be a winning strategy for player II. We define a winning strategy $\tau$ for player $K$. Let $K_{0}=\varnothing$ be $K$ 's first move, and let $L_{0}$ be $L$ 's response to $K_{0}$. We identify $L_{0}$ with player I's first move in the game $\Gamma_{1}(Y)$. Define $\tau\left(L_{0}\right)=K_{1}$ to be $\sigma\left(L_{0}\right)$.

Suppose the game $G^{*}(Y)$ has been played up to the $(n-1)$ th play $(n \geq$ 1): $K_{0}, L_{0}, \ldots, K_{n-1}, L_{n-1}$. We identify $L_{0}, \ldots, L_{n-1}$ with moves made by player $I$ in the game $\Gamma_{1}(Y)$. Define $\tau\left(L_{0}, \ldots, L_{n-1}\right)=K_{n}$ to be $\sigma\left(L_{0}, \ldots, L_{n-1}\right) \cup K_{n-1}$. Since the sequence

$$
L_{0}, \sigma\left(L_{0}\right), \ldots, L_{n-1}, \sigma\left(L_{0}, \ldots, L_{n-1}\right), \ldots
$$

is a play of the game $\Gamma_{1}(Y)$, we have $\left\{L_{n}: n<\omega\right\}$ as a discrete family. Thus, the sequence $K_{0}, L_{0}, K_{1}, L_{1}, \ldots, K_{n}, L_{n}, \ldots$, where $K_{n}=\sigma\left(L_{0}, \ldots, L_{n-1}\right)$ $\cup K_{n-1}$ for each $n$, is a winning play for player $K$. Hence, $\tau$ is a winning strategy for $K$.

To finish the proof of the theorem, let $Y$ be locally compact such that $C_{k}(Y)$ is weakly $\alpha$-favorable. By McCoy and Ntantu's theorem, player II has a winning strategy in the game $\Gamma_{1}(Y)$. By the Claim player $K$ has a winning strategy in the game $G^{*}(Y)$. By Gruenhage's theorem $Y$ is paracompact.

\section{THE $\gamma$-PROPERTY AND RELATED DEFINITIONS}

Definition 3.1. Let $\mathscr{A}$ be a collection of subsets of the space $Y$. The collection $\mathscr{A}$ is an $\omega$-cover of $Y$ means that every finite subset of $Y$ is a subset of some member of $\mathscr{A}$. A proper $\omega$-cover of $Y$ is one that does not contain $Y$. The space $Y$ has the $\gamma$-property means that for every proper open $\omega$-cover $\mathscr{A}$ of $Y$, there is an infinite $\mathscr{B} \subseteq \mathscr{A}$ such that each point of $Y$ belongs to all but finitely many sets in $\mathscr{B}$. We call $\mathscr{B}$ an eventual cover of $Y$ or an eventual subcover of $\mathscr{A}$. A subset of the real line is a $\gamma$-set if it has the $\gamma$-property.

Definition 3.2. A space $Y$ has the $\gamma^{\prime}$-property means that for each sequence of open $\omega$-covers of $Y,\left\{\mathscr{A}_{n}\right\}_{n<\omega}$, we can choose $O_{n} \in \mathscr{A}_{n}$ for each $n$ such that 
the collection $\left\{O_{n}: n<\omega\right\}$ is an eventual cover of $Y$. A subset of the real line is a $\gamma^{\prime}$-set if it has the $\gamma^{\prime}$-property.

Remark. If all finite powers of $Y$ are Lindelof, then every open $\omega$-cover of $Y$ has a countable open $\omega$-subcover [4]. Thus it suffices to consider countable open $\omega$-covers when we try to determine whether a subset of the real line is a $\gamma$-set or $\gamma^{\prime}$-set. In fact, it suffices to consider countable open $\omega$-covers consisting of elements from a given base when working with a subset of the line.

Definition 3.3. The finite-open game is played on a subset $Y$ of $\mathfrak{R}$ by two players, the finite set picker $(F)$ and the open set picker $(O)$. At the $n$th stage, $F$ chooses a finite subset $F_{n}$ of $Y$ and $O$ chooses an open subset $O_{n}$ of $Y$ such that $F_{n} \subseteq O_{n}$. The finite set picker $(F)$ wins if and only if the collection $\left\{O_{n}: n<\omega\right\}$ is an eventual cover of $Y$. Otherwise $O$ wins.

Theorem 3.4. For $Y \subseteq \Re$, the following are equivalent:

(a) $Y$ is a $\gamma$-set.

(b) $Y$ is a $\gamma^{\prime}$-set.

(c) The open set picker $(O)$ has no winning strategy in the finite-open game on $Y$.

Remark. The direction (a) $\Rightarrow$ (b) was shown in [4]. The authors in that reference claimed that (a) $\Leftrightarrow$ (c) whose proof is similar to the proof of a theorem by Sharma. Even though Sharma's theorem was stated in [4], for the sake of completeness we give the proof for $(b) \Rightarrow(c)$ and (c) $\Rightarrow(a)$.

Proof. (b) $\Rightarrow$ (c) Let $\tau$ be a strategy for the open set picker $(O)$ in the finiteopen game on $Y$. We will show that $\tau$ is not a winning strategy. First, we choose an open subset $U_{\sigma}$ for each tuple of integers $\sigma$ such that for each tuple of integers $\sigma=(i(0), \ldots, i(n))$, the collection $\mathscr{P}_{\sigma}=\left\{U_{i(0), \ldots, i(n), j}: j<\omega\right\}$ is an open $\omega$-cover of $Y$.

To start off, let $\left\{U_{n}: n<\omega\right\}$ be an $\omega$-subcover of $\{\tau(F): F \subseteq Y$ is finite $\}$. For each tuple of integers $\sigma=(i(0), \ldots, i(n))$, suppose that, for each $j \leq n$, $U_{i(0), \ldots, i(j)}$ is chosen and let $F_{0}, F_{1}, \ldots, F_{n} \subseteq Y$ be finite such that

$$
\tau\left(F_{0}, \ldots, F_{j}\right)=U_{i(0), \ldots, i(j)} \text { for each } j \leq n .
$$

Then let $\mathscr{P}_{\sigma}=\left\{U_{i(0), \ldots, i(n), j}: j<\omega\right\}$ be an $\omega$-subcover of $\left\{\tau\left(F_{0}, \ldots, F_{n}\right.\right.$, $F): F \subseteq Y$ is finite $\}$. Since there are only countably many $\mathscr{P}_{\sigma}$ 's along with the collection $\left\{U_{n}: n<\omega\right\}$, by (b) we choose one open set from each such $\omega$-cover of $Y$ and the open sets, denoted by $\left\{V_{n}: n<\omega\right\}$ where $V_{0}=U_{i(0)}$, form an eventual cover of $Y$. We can retrieve a play of the finite-open game (in which the open set picker uses $\tau$ ) by doing the following inductively:

Let $F_{0} \subseteq Y$ be finite such that $\tau\left(F_{0}\right)=U_{i(0)}=V_{0}$ and such that $\mathscr{P}_{(i(0))}$ is an $\omega$-subcover of $\left\{\tau\left(F_{0}, F\right): F \subseteq Y\right.$ is finite $\}$. Let $V_{t(1)}$ be the open set chosen from $\mathscr{P}_{(i(0))}$, and let $U_{i(0), i(1)}=V_{t(1)}$. Let $F_{1} \subseteq Y$ be finite such that $\tau\left(F_{0}, F_{1}\right)=U_{i(0), i(1)}=V_{t(1)}$ and such that $\mathscr{P}_{(i(0), i(1))}$ is an $\omega$-subcover of $\left\{\tau\left(F_{0}, F_{1}, F\right): F \subset Y\right.$ is finite $\}$. Continue this process inductively and the play of the finite-open game we obtain is one in which the open set picker loses, since $\left\{V_{n}: n \in S\right\}$ for any infinite $S \subseteq \omega$ is an eventual cover of $Y$.

(c) $\Rightarrow$ (a) Suppose $Y$ is not a $\gamma$-set. Let $\mathscr{U}$ be a countable open $\omega$-cover of $Y$ that has no eventual subcover. Then the open set picker would win if he/she chooses open sets from $\mathscr{U}$. 


\section{THE MAIN THEOREM}

Main theorem. Let $X \subseteq 2^{\omega}$. The following are equivalent.

(1) $C_{k}(T \cup X)$ is a Baire space.

(2) The space $T \cup X$ has the moving off property (MOP).

(3) $X$ is a $\gamma$-set.

(4) $X$ is a $\gamma^{\prime}$-set.

(5) The open set picker $(O)$ has no winning strategy in the finite-open game played on $X$.

(6) $C_{k}(T \cup X)$ is of second category.

We need some definitions and lemmas before proving the main theorem.

Definition 4.1. A $\pi$-base for a space $Y$ is a collection $\mathscr{B}$ of nonempty open subsets of $Y$ such that each nonempty open subset of $Y$ contains some member of $\mathscr{B}$ as a subset.

We consider the following two $\pi$-bases for $C_{k}(T \cup X)$ where $X \subseteq 2^{\omega}$. Let $\mathscr{B}_{1}$ be the collection of all nonempty basic open subsets of $C_{k}(T \cup X)$ of the form

$$
S=\left(\bigcap_{t \in T(n)}\left[\{t\}, C_{t}\right]\right) \cap\left(\bigcap_{x \in F}\left[[x \mid n+1, x], D_{x}\right]\right)
$$

where $F \subseteq X$ is finite, $C_{t}$ and $D_{x}$ are open intervals of $\mathscr{R}$, and $T(n)=$ $\bigcup_{m \leq n} 2^{m}$.

Furthermore, let $\mathscr{B}_{2}$ be the collection of all sets in $\mathscr{B}_{1}$ such that if $S \in \mathscr{B}_{2}$ and $S$ is of the same form as above, then the elements $x \mid n+1$, where $x \in F$, are all distinct. We have the following lemmas. The first one is stated without proof.

Lemma 4.2. Let $X \subseteq 2^{\omega}$. For each compact subset $Y$ of $T \cup X$, there is an integer $n$ and there is a finite set $F \subseteq X$ such that

$$
Y \subseteq T(n) \cup\left(\bigcup_{x \in F}[x \mid n+1, x]\right) .
$$

Denote the above set on the right of the set inclusion by $\mathscr{K}(n, F)$.

Lemma 4.3. The collection $\mathscr{B}_{2}$ is a $\pi$-base for $C_{k}(T \cup X)$. Hence, so is $\mathscr{B}_{1}$.

Proof. Let $V=\bigcap_{i \leq m}\left[K_{i}, U_{i}\right] \neq \varnothing$ be a basic open subset of $C_{k}(T \cup X)$.

Since $A=\bigcup_{i \leq m} K_{i}$ is compact in $T \cup X$, by Lemma 4.2 there is an integer $n$ and there is a finite set $F \subseteq X$ such that $A \subseteq \mathscr{K}(n, F)$. We can assume that $x \mid n+1, x \in F$, are all distinct. Also assume that if $[x \mid n+1, x] \cap K_{i} \neq \varnothing$ where $x \in F$, then $x \in K_{i}$. Fix $f \in \bigcap_{i \leq m}\left[K_{i}, U_{i}\right]$.

For each $x \in F$, let $D_{x}=\bigcap\left\{U_{i}: K_{i} \cap[x \mid n+1, x] \neq \varnothing\right\}$. For each $t \in T(n)$, if $t \in K_{i}$ for some $i$, let $C_{t}=\bigcap\left\{U_{i}: t \in K_{i}\right\}$. If $t$ is not in $K_{i}$ for any $i$, then let $C_{t}$ be the interval $\left(f(t)-\frac{1}{2}, f(t)+\frac{1}{2}\right)$. Let $S=\bigcap_{t \in T(n)}\left[\{t\}, C_{t}\right] \cap$ $\bigcap_{x \in F}\left[[x \mid n+1, x], D_{x}\right]$.

We claim that $S \neq \varnothing$ and $S \subseteq V$. To see the first claim, we define $h \in S$. Let $h|T(n)=f| T(n)$. Then for each $t \in T(n), h(t) \in C_{t}$. For each $x \in F$ 
let $h \mid[x \mid n+1, x]$ be constant where the constant value is in $D_{x}$. Define $h$ to be 0 on all other points. Then $h$ is continuous and $h \in S$. To see the second claim, let $g \in S$. Fix $i \leq n$ and fix $t \in K_{i}$. If $t \in T(n)$, then $g(t) \in C_{t} \subseteq U_{i}$. If $t \in[x \mid n+1, x]$ for some $x \in F$, then $g(t) \in D_{x} \subseteq U_{i}$. Thus $g\left(K_{i}\right) \subseteq U_{i}$ and the claim holds. We have shown that $\mathscr{B}_{2}$ is a $\pi$-base for $C_{k}(T \cup X)$.

Remark. The Banach-Mazur game on $C_{k}(T \cup X)$, where the empty set (E) chooses open sets from $\mathscr{B}_{2}$ and the nonempty set (NE) chooses open sets from $\mathscr{B}_{1}$, is equivalent to the Banach-Mazur game, where there is no requirement of what $\pi$-bases the open sets come from, in the sense that a player has a winning strategy in one game if and only if the same player has a winning strategy in the other game. So we assume that $\mathrm{E}$ chooses open sets from $\mathscr{B}_{2}$ and $\mathrm{NE}$ chooses open sets from $\mathscr{B}_{1}$ in the proof $(5) \Rightarrow(6)$ in the main theorem. The assumption about the $\pi$-bases will facilitate a construction in the proof of $(5) \Rightarrow(6)$.

Proof of the main theorem. (1) $\Rightarrow$ (2) See Theorem 2.2.

(3) $\Rightarrow(4),(4) \Rightarrow(5)$ See Theorem 3.4.

$(6) \Rightarrow(1)$ It is known [10] that any homogeneous space of second category is a Baire space. Since $C_{k}(Y)$ is always a homogeneous space [11], (6) $\Rightarrow(1)$ holds.

The remaining parts to be shown are $(2) \Rightarrow(3)$ and $(5) \Rightarrow(6)$.

$(2) \Rightarrow(3)$ By a remark in $\S 3$, it suffices to consider countable open $\omega$-covers consisting of elements from a given base. We use the base for $X \subseteq 2^{\omega}$ consisting of sets of the form

$$
W(B)=\{x \in X: x \mid n \in B\} \quad \text { where } B \subseteq 2^{n} \text { for some } n .
$$

Let $\left\{V_{n}: n<\omega\right\}$ be an open $\omega$-cover of $X$ such that each $V_{n}=W\left(A_{n}\right)$ for some $A_{n} \subseteq 2^{m(n)}$ (assume $A_{n} \neq 2^{m(n)}$ ). For each $n<\omega$ and for each $h>m(n)$, define

$$
A_{n, h}=\left\{f \in 2^{h}: f \supseteq g \text { for some } g \in A_{n}\right\} .
$$

Then we claim that $S=\left\{2^{h}-A_{n, h}: n<\omega, h>m(n)\right\}$ forms a moving-off collection for $T \cup X$. To see this, let $Y \subseteq T \cup X$ be compact. By Lemma 4.2, for some integer $k$ and some finite set $F \subseteq X$, we have $Y \subseteq \mathscr{K}(k, F)$. First, $F \subseteq V_{n}$ for some $n<\omega$. Let $h>\max (m(n), k+1)$. We know that, for each $x \in F, x \mid m(n) \in A_{n}$, hence $x \mid h \in A_{n, h}$. Each $[x \mid k+1, x] \cap 2^{h}$ has only one point (namely, $x \mid h$ ). Hence, for each $x \in F,\left(2^{h}-A_{n, h}\right) \cap[x \mid k+1, x]=\varnothing$ since $x \mid h \in A_{n, h}$. On the other hand, $\left(2^{h}-A_{n, h}\right) \cap T(k)=\varnothing$ since $h>k$. Thus $\left(2^{h}-A_{n, h}\right) \cap \mathscr{K}(k, F)=\varnothing$, and we show that $S$ is a moving-off collection.

By (2) we have an infinite collection $\left\{W_{n}: n<\omega\right\} \subseteq S$ that has a discrete open expansion. In particular, this means that for each $x \in X$ the branch for $x$ in the Cantor tree misses $W_{n}$ eventually, i.e., for all but finitely many $n(*)$.

For each $n<\omega$, we know $W_{n}=2^{h(n)}-A_{t(n), h(n)}$ for some $t(n)<\omega$ and for some $h(n)>m(t(n))$, and we let $U_{n}=V_{t(n)}$ (note that $\left.V_{t(n)}=W\left(A_{t(n)}\right)\right)$. We claim for each $x \in X, x \in U_{n}$ eventually. By knowing $(*)$ above, we see that the branch for $x$ meets $A_{t(n)}$ eventually and hence belongs to $V_{t(n)}$ eventually for each $x \in X$. 
(5) $\Rightarrow(6)$ Let $X \subseteq 2^{\omega}$. Define $[X]^{<\omega}=\{F \subseteq X: F$ is finite $\}$ and define the functions

$$
\mathscr{N}: \mathscr{B}_{1} \rightarrow \omega, \quad \mathscr{F}: \mathscr{B}_{1} \rightarrow[X]^{<\omega},
$$

and $\mathscr{C}(t, S)$ and $\mathscr{D}(x, S)$ by letting, for each

$$
S=\bigcap_{t \in T(n)}\left[\{t\}, C_{t}\right] \cap \bigcap_{x \in F}\left[[x \mid n+1, x], D_{x}\right] \in \mathscr{B}_{1},
$$

$\mathscr{N}(S)=n, \mathscr{F}(S)=F$, and, for each $t \in T(n)$ and for each $x \in F, \mathscr{C}(t, S)=$ $C_{t}$ and $\mathscr{D}(x, S)=D_{x}$.

By the remark in $\S 2$ about the game characterization for second category, it suffices to show that the empty set player has no winning strategy in the game $\mathrm{BM}\left(C_{k}(T \cup X)\right)$ with NE making the first move. Let $\sigma$ be a strategy for $\mathrm{E}$ and we show $\sigma$ is not a winning strategy. Before we do so, we define the following:

(i) a function $A\left(V_{0}, \ldots, V_{n}\right)$,

(ii) a counterstrategy $\tau$ for $\mathrm{NE}$,

(iii) a strategy $\theta$ for the open set picker in the finite-open game on $X$.

(i) For each partial play of the Banach-Mazur game on $C_{k}(T \cup X), V_{0}$, $U_{1}, V_{1}, \ldots, U_{n}, V_{n}, U_{n+1}$, where for each $i$ with $1 \leq i \leq n+1, U_{i}=$ $\sigma\left(V_{0}, \ldots, V_{i-1}\right)$, we define

$$
A\left(V_{0}, \ldots, V_{n}\right)=\left\{f \in 2^{b}: f=x \mid b \text { for some } x \in \mathscr{F}\left(V_{n}\right)\right\}
$$

where $b-1=\mathscr{N}\left(U_{n+1}\right)$.

(ii) We define $\tau$ that depends on E's last move and a finite subset of $X$. Suppose that $U_{h+1}$ is the empty set's last move and $F \subseteq X$ is finite.

Consider $n=\mathscr{N}\left(U_{h+1}\right)$ and $G=\mathscr{F}\left(U_{h+1}\right)$. We define NE's next move $\tau\left(U_{h+1}, F\right)=V_{h+1}$. For a technical reason which will make a subsequent construction tidier, we would like the empty set's response to $V_{h+1}, U_{h+2}=$ $\sigma\left(V_{0}, \ldots, V_{h+1}\right)$, to satisfy $n=\mathscr{N}\left(V_{h+1}\right)<\mathscr{N}\left(U_{h+2}\right)$. To this end we take the following step:

Choose $p$ and $q$ (with $p \neq q$ ) such that $p|n+1=q| n+1$, and let $G(1)=$ $G \cup F \cup\{p, q\}$. We now define $\tau\left(U_{h+1}, F\right)=V_{h+1}$ of the form

$$
V_{h+1}=\bigcap_{t \in T(n)}\left[\{t\}, C_{t}\right] \cap \bigcap_{x \in G(1)}\left[[x \mid n+1, x], D_{x}\right]
$$

by the steps $(\mathrm{A})-(\mathrm{D})$.

(A) For each $t \in T(n)$, let length $\left(C_{t}\right)<2^{-(h+1)}$ and $\mathrm{CL}\left(C_{t}\right) \subseteq \mathscr{C}\left(t, U_{h+1}\right)$. Note that $\mathrm{CL}$ is the closure operator.

(B) For each $x \in G$, let length $\left(D_{x}\right)<2^{-(h+1)}$ and $\operatorname{CL}\left(D_{x}\right) \subseteq \mathscr{D}\left(x, U_{h+1}\right)$.

(C) For each $y \in G(1)-G$ such that $[y \mid n+1, y] \cap[x \mid n+1, x] \neq \varnothing$ for some $x \in G$, let $D_{y}=D_{x}$.

(D) For each $y \in G(1)-G$ such that $[y \mid n+1, y] \cap[x \mid n+1, x]=\varnothing$ for all $x \in G$, let $D_{y}=\left(-2^{-(h+1)}, 2^{-(h+1)}\right)$.

Note that it is easy to verify that $V_{h+1} \neq \varnothing$.

(iii) We now define a strategy $\theta$ for the open set picker. To start off, let $F_{0} \subseteq X$ be finite and $V_{0}=\bigcap_{x \in G}\left[B_{x},\left(-\frac{1}{2}, \frac{1}{2}\right)\right]$ where $B_{x}=\{x \mid n: n<\omega\} \cup\{x\}$ for $x \in G=F_{0}$. Let $U_{1}=\sigma\left(V_{0}\right)$ and define $\theta\left(F_{0}\right)=W\left(A\left(V_{0}\right)\right.$ ) (see the proof 
TABLE 1

\begin{tabular}{|cccc|}
\hline$F$ & $\mathrm{NE}$ & $\mathrm{E}$ & $O$ \\
\hline$F_{0}$ & $V_{0}$ & $U_{1}$ & $\theta\left(F_{0}\right)=O_{0}$ \\
$F_{1}$ & $V_{1}=\tau\left(U_{1}, F_{1}\right)$ & $U_{2}=\sigma\left(V_{0}, V_{1}\right)$ & $\theta\left(F_{0}, F_{1}\right)=O_{1}=W\left(A\left(V_{0}, V_{1}\right)\right)$ \\
$\vdots$ & $\vdots$ & $\vdots$ & $\vdots$ \\
$\dot{F_{k}}$ & $V_{k}=\tau\left(U_{k}, F_{k}\right)$ & $U_{k+1}=\sigma\left(V_{0}, \ldots, V_{k}\right)$ & $\theta\left(F_{0}, \ldots, F_{k}\right)=O_{k}=W\left(A\left(V_{0}, \ldots, V_{k}\right)\right)$ \\
$\vdots$ & $\vdots$ & $\vdots$ & $\vdots$ \\
\hline
\end{tabular}

for $(2) \Rightarrow(3)$ for the notation $W)$. The remaining definition of $\theta$ is in Table 1.

We are now back to the task of showing $\sigma$ is not a winning strategy for empty set player (E) in the game $\operatorname{BM}\left(C_{k}(T \cup X)\right)$. By (5) the strategy $\theta$ we just defined is not winning for $O$. Hence there is a play of the finite-open game with $O$ using the strategy $\theta$ that defeats $O$. We use the table in (iii) to denote this play of the finite-open game and claim that the play of the Banach-Mazur game, in which $\mathrm{E}$ makes his/her moves by the strategy $\sigma$ that is exhibited in the same table, is one that defeats $E$. To this end we define a continuous function $H: T \cup X \rightarrow \mathbb{R}$ such that $H \in \bigcap_{j<\omega} V_{j}$.

Note. For each $x \in X$, there is some $k<\omega$ such that $x \in O_{j}$ for all $j \geq$ $k$. For each $n$ let $A_{n}=A\left(V_{0}, \ldots, V_{n}\right)$ and note that $O_{n}=W\left(A_{n}\right)$. From definition (i) we see that $A_{n} \subseteq 2^{m(n)}$ where $m(n)-1=\mathscr{N}\left(U_{n+1}\right)$. Then, if $x \in O_{n}, x \mid m(n) \in A_{n}$. Also, $\{m(n)\}_{n<\omega}$ is an increasing sequence of integers (see (ii)).

For each $t \in T$ let $H(t)$ be the number in the intersection of all open intervals $\mathscr{C}\left(t, V_{j}\right)$ and $\mathscr{C}\left(t, U_{j}\right)$. We show that $H: T \rightarrow \mathbb{R}$ can be extended to $H: T \cup X \rightarrow \mathbb{R}$.

Let $x \in X$. There is some integer $k$ such that $x \in O_{n}$ for all $n \geq k$. Let $a_{n}=x \mid m(n)$ for each $n \geq k$. Since $a_{n} \in A_{n}$ for each $n \geq k$, we have $a_{n}=x_{n} \mid m(n)$ for some $x_{n} \in \mathscr{F}\left(V_{n}\right)$. For each $n>k$, let $D_{n}=\mathscr{D}\left(x_{n}, V_{n}\right)$, $E_{n}=\mathscr{D}\left(x_{n}, U_{n+1}\right)$, and we know that $\operatorname{length}\left(D_{n}\right)<2^{-n}$. It is clear that $E_{n} \subseteq D_{n}$ since $U_{n+1} \subseteq V_{n}$. We claim the following:

Claim 1. For each $n>k, \operatorname{CL}\left(D_{n+1}\right) \subseteq E_{n} \subseteq D_{n}$.

Proof. We know that $a_{n}=x_{n}\left|m(n), a_{n+1}=x_{n+1}\right| m(n+1)$, and $a_{n} \subseteq a_{n+1}$. Thus, we have $x_{n}\left|m(n) \subseteq x_{n+1}\right| m(n+1)$, which implies $x_{n}\left|m(n)=x_{n+1}\right| m(n)$. In showing $\mathrm{CL}\left(D_{n+1}\right) \subseteq E_{n}$ we consider two cases:

Case 1. $x_{n+1} \in \mathscr{F}\left(U_{n+1}\right)$. We know $x_{n} \in \mathscr{F}\left(U_{n+1}\right)$. Since $U_{n+1} \in \mathscr{B}_{2}$, the elements $y \mid m(n), y \in \mathscr{F}\left(U_{n+1}\right)$, are all distinct. If $x_{n+1} \neq x_{n}$, then $x_{n}\left|m(n) \neq x_{n+1}\right| m(n)$ (which is not true). Thus $x_{n+1}=x_{n}$ and $\mathscr{D}\left(x_{n}, V_{n+1}\right)$ $=\mathscr{D}\left(x_{n+1}, V_{n+1}\right)=D_{n+1}$. In defining $V_{n+1}$ (in condition (B) of definition (ii)), for each $y \in \mathscr{F}\left(U_{n+1}\right), \operatorname{CL}\left(\mathscr{D}\left(y, V_{n+1}\right)\right)$ was chosen to be a subset of $\mathscr{D}\left(y, U_{n+1}\right)$. Thus $\operatorname{CL}\left(\mathscr{D}\left(x_{n}, V_{n+1}\right)\right) \subseteq \mathscr{D}\left(x_{n}, U_{n+1}\right)$, and this set-inclusion is the desired one: $\mathrm{CL}\left(D_{n+1}\right) \subseteq E_{n}$.

Case 2. $x_{n+1} \notin \mathscr{F}\left(U_{n+1}\right)$. We have $x_{n} \in \mathscr{F}\left(U_{n+1}\right)$ and $x_{n+1} \in \mathscr{F}\left(V_{n+1}\right)-$ $\mathscr{F}\left(U_{n+1}\right)$. Since $x_{n}\left|m(n)=x_{n+1}\right| m(n),\left[x_{n} \mid m(n), x_{n}\right] \cap\left[x_{n+1} \mid m(n), x_{n+1}\right] \neq$ $\varnothing$. So $D_{n+1}$ is chosen to be the same as $\mathscr{D}\left(x_{n}, V_{n+1}\right)$ (see condition (C) in definition (ii)). Furthermore, $\operatorname{CL}\left(\mathscr{D}\left(x_{n}, V_{n+1}\right)\right) \subseteq \mathscr{D}\left(x_{n}, U_{n+1}\right)=E_{n}$. 
Claim 2. For each $n>k, H\left(\left[a_{n}, a_{n+1}\right)\right) \subseteq D_{n+1}$ where $\left[a_{n}, a_{n+1}\right)=\{t \in$ $T: a_{n} \subseteq t \subseteq a_{n+1}$ and $\left.t \neq a_{n+1}\right\}$.

Proof. Note that $\mathcal{N}\left(V_{n+1}\right)=\mathscr{N}\left(U_{n+1}\right)=m(n)-1$ and $\mathcal{N}\left(U_{n+2}\right)=$ $m(n+1)-1$, and in definition (ii) we designed $m(n)<m(n+1)$. Furthermore, $a_{n}=x_{n} \mid m(n)$ and $a_{n+1}=x_{n+1} \mid m(n+1)$. For each $t \in\left[a_{n}, a_{n+1}\right)$, $t \in T\left(\mathscr{N}\left(U_{n+2}\right)\right)$ and $\mathscr{C}\left(t, U_{n+2}\right) \subseteq D_{n+1}$ since $U_{n+2} \subseteq V_{n+1} . H(t) \in D_{n+1}$ since $H(t) \in \mathscr{C}\left(t, U_{n+2}\right)$.

By Claims 1 and 2 it is now easy to see that $\{H(x \mid j)\}_{j<\omega}$ is a convergent sequence of numbers, and we let $H(x)$ be the limit of the sequence. The function $H$ is continuous, and our task now is to show $H \in \bigcap_{j<\omega} V_{j}$.

Remark. If $x \in \bigcup_{n<\omega} \mathscr{F}\left(V_{n}\right)$, then the $x_{n}$ 's in the argument above are all $x$ and the $H(x)$ just defined belongs to $\mathscr{D}\left(x_{n}, V_{n}\right)=D_{n}$ for each $n>k$.

Finally we claim that $H \in V_{j}$ for each $j<\omega$. Fix $j$ and let $n=\mathscr{N}\left(V_{j}\right)$ and $G=\mathscr{F}\left(V_{j}\right)$. For each $t \in T(n)$, we know that $H(t) \in \mathscr{C}\left(t, V_{j}\right)$ because $H(t)$ is chosen to be in the intersection of all such intervals. For each $x \in G$, we need to show

$$
H([x \mid n+1, x]) \subseteq \mathscr{D}\left(x, V_{j}\right)
$$

For each $t \in[x \mid n+1, x)$, there is some $i>j$ such that $t \in T\left(\mathscr{N}\left(U_{i}\right)\right)$. Since $U_{i} \subseteq V_{j}, \mathscr{C}\left(t, U_{i}\right) \subseteq \mathscr{D}\left(x, V_{j}\right)$. Hence $H(t) \in \mathscr{D}\left(x, V_{j}\right)$ because $H(t) \in$ $\mathscr{C}\left(t, U_{i}\right)$. Note that $\mathscr{D}\left(x, V_{q}\right) \subseteq \mathscr{D}\left(x, V_{j}\right)$ for all $q>j$. By the remark above, $H(x) \in \mathscr{D}\left(x, V_{q}\right)$ for all $q>k$ for some $k$. Thus we have $H(x) \in$ $\mathscr{D}\left(x, V_{j}\right)$. So we have shown that for each $t \in T(n), H(t) \in \mathscr{C}\left(t, V_{j}\right)$, and, for each $x \in \mathscr{F}\left(V_{j}\right), H([x \mid n+1, x]) \subseteq \mathscr{D}\left(x, V_{j}\right)$. This shows $H \in V_{j}$.

\section{EXAMPLES}

Example 5.1 $(\mathrm{MA}+\neg \mathrm{CH})$. Under MA, every subset of the real line of cardinality < continuum is a $\gamma$-set [4]. Let $X \subseteq 2^{\omega}$ be uncountable with $|X|<$ continuum. By the main theorem $C_{k}(T \cup X)$ is a Baire space, while $T \cup X$ is not paracompact but normal [14, Example $\left.\mathrm{G}^{\prime}\right]$.

Example 5.2 (MA). There is a $\gamma$-set of cardinality continuum under MA [3]. Let $X \subseteq 2^{\omega}$ be such a set. By the main theorem $C_{k}(T \cup X)$ is a Baire space. Note that $X$ is a closed discrete subset of $T \cup X$ and that any separable space with a closed discrete subset of cardinality continuum is not normal. It follows that $T \cup X$ is not normal.

Consider $C_{i}=\left\{f \in 2^{\omega}: f(0)=i\right\}, X_{i} \subseteq C_{i}$ for $i=0,1$. Furthermore, for each $i=0,1$, consider

$$
Y_{i}=\left\{f \in 2^{\omega}: \text { for some } g \in X_{i}, f(n)=g(n+1) \text { for all } n<\omega\right\} .
$$

Note that each $Y_{i}$ is homeomorphic to $X_{i}$. It is easy to see that

$$
T \cup\left(X_{0} \cup X_{1}\right) \cong\{\varnothing\} \oplus\left(T \cup Y_{0}\right) \oplus\left(T \cup Y_{1}\right) \text {. }
$$

Furthermore, it is known [12] that $C_{k}\left(\bigoplus_{\alpha \in S} X_{\alpha}\right) \cong \prod_{\alpha \in S} C_{k}\left(X_{\alpha}\right)$. Thus we have

$$
C_{k}\left(T \cup X_{0} \cup X_{1}\right) \cong \mathbb{R} \times C_{k}\left(T \cup Y_{0}\right) \times C_{k}\left(T \cup Y_{1}\right)
$$

Example 5.3. Todorcevic (quoted in $[2,3]$ ) proved that, in some models of set theory, there are subsets $X_{0}$ and $X_{1}$ of the line that are $\gamma$-sets, but $X_{0} \cup X_{1}$ 
is not a $\gamma$-set. Consider two such sets and their homeomorphic copies in $C_{0}$ and $C_{1}$ respectively. Say $X_{0} \subseteq C_{0}$ and $X_{1} \subseteq C_{1}$. We have ( $\left.\sharp\right)$. Note that the left-hand side of $(\sharp)$ is not a Baire space. The product of two Baire spaces is a Baire space if one of them is weakly $\alpha$-favorable [7, Theorem 5.1]. Thus $\mathbb{R} \times Z$ is a Baire space whenever $Z$ is Baire, and it follows that $C_{k}\left(T \cup Y_{0}\right) \times C_{k}\left(T \cup Y_{1}\right)$ is not a Baire space while both $C_{k}\left(T \cup Y_{0}\right)$ and $C_{k}\left(T \cup Y_{1}\right)$ are Baire spaces.

\section{ACKNOWLEDGMENT}

The author is deeply grateful for the encouragement and helpful suggestions from Professor G. Gruenhage, especially for bringing to the author's attention the existence of the two $\gamma$-sets for Example 5.3.

\section{REFERENCES}

1. W. G. Fleissner and K. Kunen, Barely Baire spaces, Fund. Math. 101 (1978), 229-240.

2. D. H. Fremlin and A. W. Miller, On some properties of Hurewicz, Menger, and Rothberger, Fund. Math. 129 (1988), 17-33.

3. F. Galvin and A. W. Miller, $\gamma$-sets and other singular sets of real numbers, Topology Appl. 17 (1984), 145-155.

4. J. Gerlits and Zs. Nagy, Some properties of $C(X)$. I, Topology Appl. 14 (1982), 151-161.

5. J. Gerlits, Some properties of $C(X)$. II, Topology Appl. 15 (1983), 255-262.

6. G. Gruenhage, Games, covering properties and Eberlein compacts, Topology Appl. 23 (1986), 291-297.

7. R. C. Haworth and R. A. McCoy, Baire spaces, Dissertationes Math. (Rozprawy Mat.) 141 (1977), 1-77.

8. K. Kunen, Set theory, An Introduction to Independent Proofs, North-Holland, Amsterdam.

9. R. Laver, On the consistency of Borel's conjecture, Acta Math. 137 (1976), 151-169.

10. D. J. Lutzer and R. A. McCoy, Category in function spaces. I, Pacific J. Math. 90 (1980), 145-168.

11. R. A. McCoy and I. Ntantu, Completeness properties of function spaces, Topology Appl. 22 (1986), 191-206.

12. __ Topological properties of spaces of continuous functions, Lecture Notes in Math., vol. 1315, Springer-Verlag, New York.

13. E. G. Pytkeev, Baire property of spaces of continuous functions, Mat. Zametki 38 (1985), 726-740.

14. F. D. Tall, Normality versus collectionwise normality, Handbook of Set-Theoretic Topology, North-Holland, Amsterdam, 1984.

15. J. van Mill and R. Pol, The Baire category theorem in products of linear spaces and topological groups, Topology Appl. 22 (1986), 267-282.

16. H. E. White, Topological spaces that are $\alpha$-favorable for a player with perfect information, Proc. Amer. Math. Soc. 50 (1975), 477-482.

17. S. Willard, General topology, Addison-Wesley, Reading, MA, 1970.

Division of Mathematics, AUburn University, Auburn University, Alabama 36849 